\title{
Numerical Simulation of Wind Turbine Aerodynamic Characteristics under Wind Shear Based on Lattice-Boltzmann Method
}

\author{
Chengdong Yuan ${ }^{1}$, Jinlong Wang ${ }^{2}$, Yueyue Pan $^{2, *}$, Hui Chen ${ }^{1}$ and Xiaqi Zhang ${ }^{1}$ \\ ${ }^{1}$ Shandong university of science and technology, Shandong, China, 266590 \\ ${ }^{2}$ Weifang university, Shandong, China, 261061
}

\begin{abstract}
In order to study the influence of wind shear on the aerodynamic characteristics of large wind turbines, taking the 5MW wind turbine blade model published by NREL as the research object, large eddy simulation (LES) of wind turbines was carried out by using XFlow fluid simulation software based on LatticeBoltzmann method (LBM). WALE turbulence model was used to study wind shear at 3, 11.2 and $25 \mathrm{~m} / \mathrm{s}$ wind speeds. The effect of factors on the axial thrust and torque of wind turbines is compared with the data published by NREL. The results show that the XFlow software based on LBM and LES method has good capturing ability for the eddy wake of wind turbine; wind shear causes the airfoil section of each section of blade to deviate from the best designed attack angle in theory and results in a decrease in torque applied to the wind turbine.
\end{abstract}

\section{Introduction}

Under the trend of large-scale development of wind turbines, it becomes particularly important to accurately analyze the aerodynamic performance of large wind turbines. According to the aerodynamic loads of wind turbines obtained from the analysis, the aerodynamic layout optimization design of wind turbines is carried out, which ensures the structural strength and at the same time obtains a higher wind energy utilization coefficient [1].At present, experimental and numerical simulation methods are mainly used to study the aerodynamic performance of wind turbines. The numerical simulation methods mainly include Blade-element Theory (BEM), eddy current theory and computational fluid dynamics method (CFD) [2]. With the improvement of computer performance, large commercial CFD software has been more and more widely used and has become a sharp tool for wind turbine aerodynamic performance research [3]. This paper takes

Table 1. Main parameters of NREL5 MW wind turbine

\begin{tabular}{|c|c|c|c|}
\hline Parameters & Numerical value & Parameters & Numerical value \\
\hline Rating & $5 \mathrm{MW}$ & Cut-in wind speed & $3 \mathrm{~m} / \mathrm{s}$ \\
\hline The number of blades & 3 & Cut-out wind speed & $25 \mathrm{~m} / \mathrm{s}$ \\
\hline Wind turbine diameter & $123 \mathrm{~m}$ & Rated wind speed & $11.4 \mathrm{~m} / \mathrm{s}$ \\
\hline Hub diameter & $3 \mathrm{~m}$ & Cut-in rotor speed & $6.9 \mathrm{r} / \mathrm{min}$ \\
\hline Hub height & $90 \mathrm{~m}$ & Rated rotor speed & $12.1 \mathrm{r} / \mathrm{min}$ \\
\hline Shaft tilt & $5^{\circ}$ & Cone angle & $2.5^{\circ}$ \\
\hline
\end{tabular}

\footnotetext{
*Corresponding author e-mail: panyueyue1022@wfu.edu.cn; panyueyue_1022@163.com
}

\subsection{Three-dimensional model of wind turbine}

This paper takes NREL $5 \mathrm{MW}$ wind turbine as the research object. The wind turbine is a horizontal axis, three-blade and windward wind power unit [4]. Its main parameters are shown in Table 1. Laboratory (NREL) as the research object, large eddy XFlow fluid simulation software, and studies the influence of wind shear factor on axial thrust and torque of wind turbine at 3, 11.2 and $25 \mathrm{~m} / \mathrm{s}$ wind speeds, and verifies the reliability of simulation software by comparing it with published data of NREL.

\section{models and computational domains}

5MW wind turbine of National Renewable Energy 
In this paper, the numerical simulation calculation model only considers the wind turbine part, without considering the influence of engine compartment and tower. The wind turbine has elevation and cone angle. The wind turbine model is shown in Figure 1.

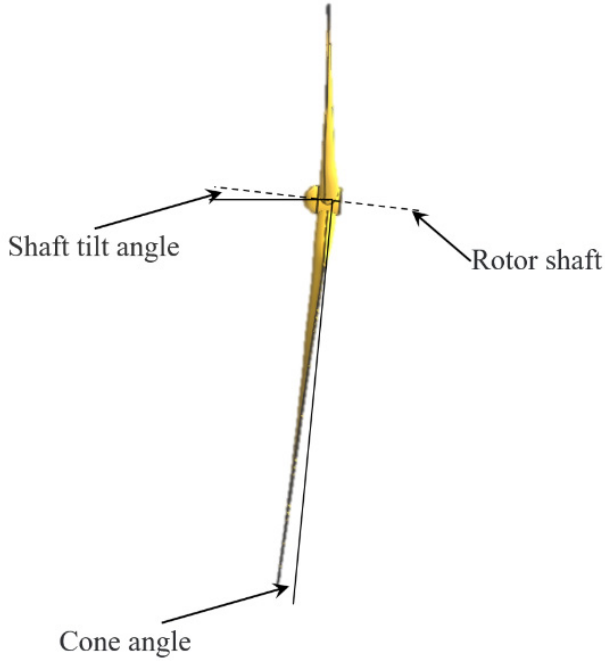

Figure 1. Geometric model of wind turbine

\subsection{Wind field modeling and meshing}

After verification of irrelevance of wind field size, the selected wind field size is $600 \mathrm{~m} \times 290 \mathrm{~m} \times 400 \mathrm{~m}$.
Considering the influence of ground, the height of wind wheel center from ground is $90 \mathrm{~m}$, and the distance from inlet of inflow is $200 \mathrm{~m}$. Both left and right boundary and upper boundary of calculation area are $200 \mathrm{~m}$, as shown in Figure 2.

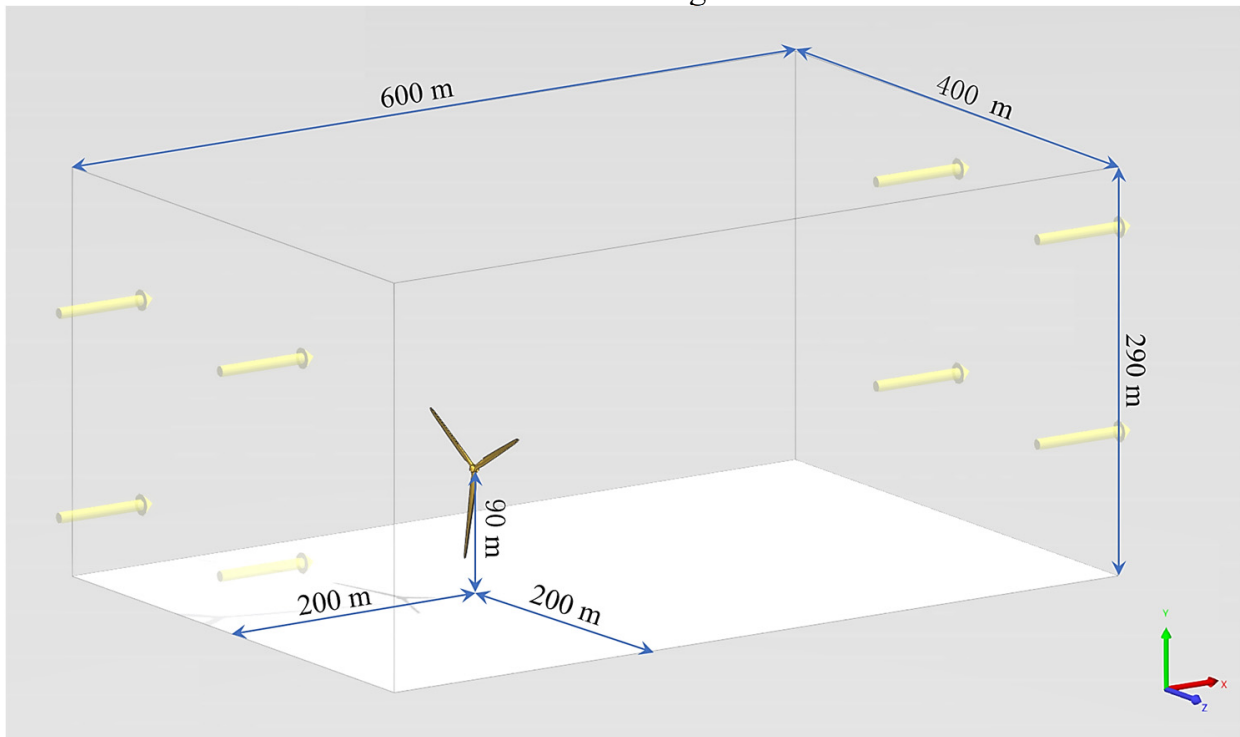

Figure 2. calculation domain of wind field

\section{CFD software principles and numerical methods}

\subsection{Introduction to XFlow software}

XFlow is a new generation of fluid dynamics simulation analysis software based on Lattice-Boltzmann method (LBM). It uses particle-based and complete Lagrange function method, has advanced large eddy simulation (LES) model and uses uniform non-equilibrium wall function to simulate boundary layer.

\subsection{LBM Method}

The LBM method is to discrete the Boltzmann-BGK equation in space, time and velocity [5]. The Boltzmann transport equation is as follows:

$f_{i}\left(r+c_{i} \Delta t, t+\Delta t\right)=f_{i}(r, t)+\Omega_{i}^{B}\left(f_{1}, \ldots, f_{b}\right)$

Where $f_{i}$ is the distribution function in the direction; $\Omega_{i}^{B}$ is the collision operator; $t$ is the discrete time, s; $c_{i}$ is the velocity in the $i$ direction, $\mathrm{m} / \mathrm{s} ; r$ is the 
position on the lattice.

The lattice Boltzmann method (LBM) defines a simplified operator under the Bhatnagar-Gross-Krook (BGK) [6] approximation as follows:

$$
\Omega_{i}^{B G K}=\frac{1}{\tau}\left(f_{i}^{e q}-f_{i}\right)
$$

Where $f_{i}^{e q}$ is a local equilibrium function; $\tau$ is a relaxation characteristic time.

Typically, the balanced distribution function expression is as follows:

$$
f_{i}^{e q}(r, t)=t_{i} \rho\left(1+\frac{c_{i a} v_{a}}{c_{s}^{2}}+\frac{v_{\alpha} v_{\beta}}{2 c_{s}^{2}}\left(\frac{c_{i \alpha} c_{i \beta}}{c_{s}^{2}}-\delta_{\alpha \beta}\right)\right)
$$

Where $c^{s}$ is the sound velocity, $\mathrm{m} / \mathrm{s} ; v_{\alpha}$ and $v_{\beta}$ are the macro-viscosities, Pa.s; $\delta_{\alpha \beta}$ is the Croneker function; $t_{i}$ is the parameter to ensure spatial isotropy; $\rho$ is the macro-density, $\mathrm{kg} / \mathrm{m} 3$.

\section{3 large eddy simulation Method}

Large eddy simulation method (LES) filters continuity equation and $\mathrm{N}-\mathrm{S}$ equation, filters subgrid vortices smaller than filter scale, decomposes control equation describing the motion of large eddy, and introduces subgrid stress term into the equation to consider the influence of momentum and energy of subgrid vortices on large eddy [7]. The subgrid turbulence model used in XFlow software is a wall-adaptive local eddy viscosity model (WALE), which has good characteristics in both laminar and turbulent flows, whether close to the wall or far from the wall. The model restores the asymmetric characteristics of the turbulent boundary layer without adding artificial turbulent viscosity to the wake shear zone, which can be directly solved. The WALE model [8] is represented as follows:

$$
\begin{aligned}
& \mu_{\mathrm{t}}=\rho L_{\mathrm{s}}{ }^{2} \frac{\left(S_{i j}^{d} \cdot S_{i j}^{d}\right)^{3 / 2}}{\left(\bar{S}_{i j}^{d} \cdot \bar{S}_{i j}^{d}\right)^{5 / 2}+\left(S_{i j}^{d} \cdot S_{i j}^{d}\right)^{5 / 4}} \\
& L_{s}=\min \left(\kappa d, C_{w} V^{1 / 3}\right)
\end{aligned}
$$

$S_{i j}^{d}=\frac{1}{2}\left(\bar{g}_{i j}^{2}+\bar{g}_{i j i}^{2}\right)$

Where WALE constant $C_{w}$ is usually $0.2 ; S_{i j}^{d}$ is the strain rate tensor, which reflects not only the function of the symmetric part of the velocity gradient tensor, but also the effect of the asymmetric part.

\subsection{Wind shear model}

The NREL 5 MW large wind turbine is $123 \mathrm{~m}$ in diameter and the effects of wind shear must be taken into account. Changes in wind speed perpendicular to the wind plane are called wind shear or wind speed profiles, where an exponential law distribution is used, as shown in the Equation (8):

$$
U=U_{\text {ref }}\left(\frac{y}{y_{\text {ref }}}\right)^{\alpha}
$$

In the formula, $U$ is the average wind speed at $y$ above ground level, $\mathrm{m} / \mathrm{s} ; y_{\text {ref }}$ is the reference height, taking the hub center height of $90 \mathrm{~m}$; $U_{r e f}$ is the average wind speed at the hub center height, $\mathrm{m} / \mathrm{s} ; \alpha$ is the wind speed profile index, and here take $1 / 7$.

\section{Numerical simulation results and analysis}

Under three wind speeds of $3 \mathrm{~m} / \mathrm{s}, 11.2 \mathrm{~m} / \mathrm{s}$ and $25 \mathrm{~m} / \mathrm{s}$, the wind turbine is simulated under two conditions of wind shear and no wind shear, and the axial thrust and torque changes of the wind turbine are monitored. The lattice analytical scale of calculation domain is $4 \mathrm{~m}$ for far-field solution, $0.25 \mathrm{~m}$ for near-wall solution and $0.25 \mathrm{~m}$ for eddy wake solution. Dynamic adaptive tracking encryption control is used for eddy wake. At this time, the number of grids is about 19.32 million and the calculation time is about 15 hours. The calculation accuracy and cost of eddy wake simulation are relatively balanced. The lattice analytical scale is shown in Figure 3 and the threedimensional volume field cloud of vorticity is shown in Figure 4. 


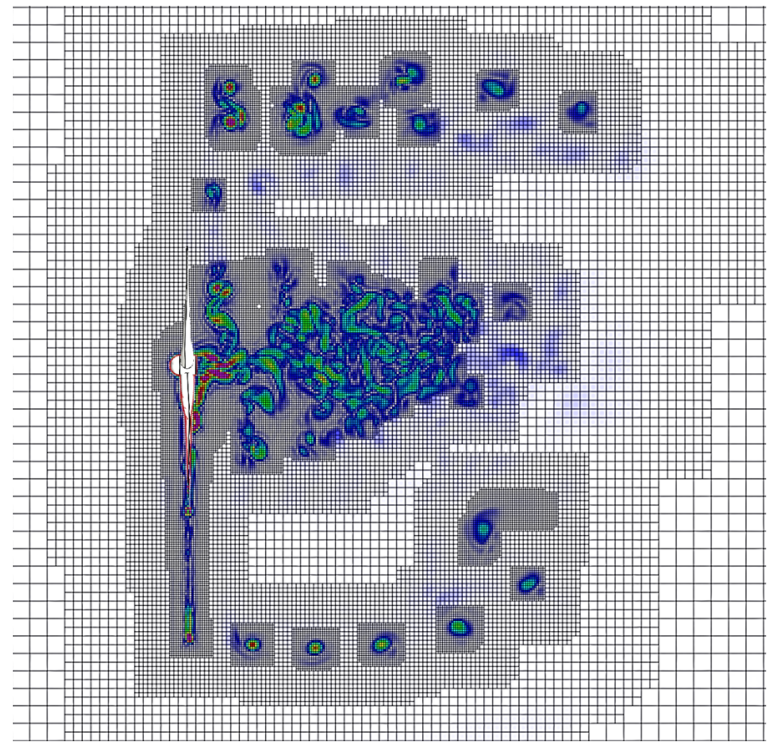

Figure 3. The lattice analytical scale

It can be seen from Figure 3 that obvious vortex wake is formed behind the blade, and the grid analytical scale in the wake area is automatically adjusted. From Figure 4, it can be seen that the spiral vortex wake is formed in the wind field due to the rotation of the wind turbine, and vortices of different sizes are generated in the wake, which can well simulate the flow state of subgrid vortex. In other words, XFlow software can automatically detect and capture the large and sub-grid vortices in the wake area,

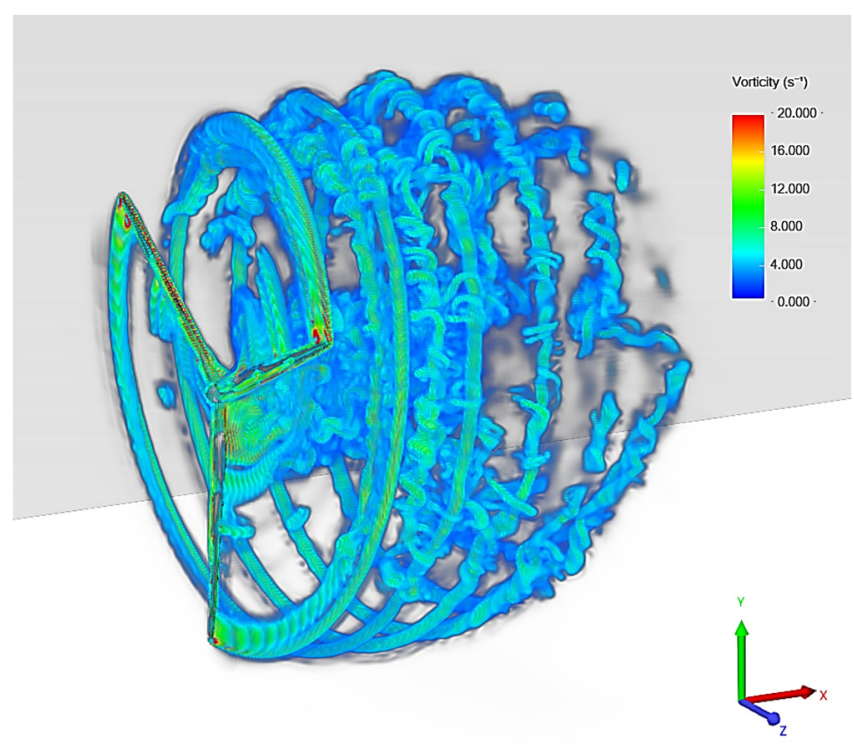

Figure 4. The 3D volume field cloud of vorticity

update and encrypt the grid analytical scale of the wake area in real time, accurately simulate the vortex wake of wind turbine, and effectively save computing resources.

The data of numerical simulation results select the average load value within 5 rotation cycles after stabilization and compare with NREL published data, as shown in Table 2, where $\mathrm{F}_{\mathrm{XB}}$ is the axial thrust of the wind turbine, and $\mathrm{M}_{\mathrm{XB}}$ is the torque of the wind turbine.

Table 2. Comparison of simulation data and NREL data

\begin{tabular}{|c|c|c|c|c|c|c|c|}
\hline \multicolumn{2}{|c|}{ Operating conditions } & \multicolumn{6}{|c|}{ Wind turbine load } \\
\hline $\begin{array}{c}\text { Wind speed } \\
(\mathrm{m} / \mathrm{s})\end{array}$ & Wind shear & $\begin{array}{l}\mathrm{F}_{\mathrm{XB}} \\
(\mathrm{kN})\end{array}$ & $\begin{array}{c}\text { F XB } \text { of NREL }_{(\mathrm{kN})} \\
\end{array}$ & $\begin{array}{c}\text { Error } \\
(\%)\end{array}$ & $\begin{array}{c}\mathrm{M}_{\mathrm{XB}} \\
(\mathrm{kN} \cdot \mathrm{m})\end{array}$ & $\begin{array}{c}\mathrm{M}_{\mathrm{XB}} \text { of NREL } \\
(\mathrm{kN} \cdot \mathrm{m})\end{array}$ & $\begin{array}{c}\text { Error } \\
(\%)\end{array}$ \\
\hline \multirow{2}{*}{3} & Yes & 149 & 168 & $11 \%$ & 58 & 56 & $4 \%$ \\
\hline & No & 155 & 168 & $8 \%$ & 61 & 56 & $9 \%$ \\
\hline \multirow{2}{*}{11.4} & Yes & 760 & 794 & $4 \%$ & 4318 & 4181 & $3 \%$ \\
\hline & No & 766 & 794 & $4 \%$ & 4362 & 4181 & $4 \%$ \\
\hline \multirow{2}{*}{25} & Yes & 345 & 373 & $8 \%$ & 4320 & 4181 & $3 \%$ \\
\hline & No & 350 & 373 & $6 \%$ & 4370 & 4181 & $5 \%$ \\
\hline
\end{tabular}

According to the data comparison in Table 2, under all working conditions, the axial thrust of the wind turbine is slightly less than the NREL open value, and the wind turbine torque is slightly greater than the NREL open value, and the error at cut-in wind speed is relatively large, and the error at rated wind speed is relatively small. The results show that the relative errors between the numerical simulation results and NREL open results of the axial force and the torque of the wind turbine are within a reasonable range, which shows that the XFlow software based on Lattice-Boltzmann method and large eddy simulation has high accuracy for the simulation of wind turbine vortex wake. In addition, compared with the effect of wind shear, it is found that the thrust and torque of the rotor shaft under the action of wind shear are relatively less than the value of no wind shear under the corresponding wind speed. This is because the wind shear causes the wind speed of the wind turbine blade to be larger when it is at the high position in the rotation cycle, and the wind speed is small when it is at the low position, and the incoming wind speed of the blade presents periodic fluctuations, which deviates from the theoretical design wind speed. The results show that the angle of attack of each airfoil periodically deviates from the optimal design angle of attack, resulting in the reduction of wind turbine torque, which is less than the value without wind shear.

\section{Conclusion}

(1) The XFlow software based on LBM and LES method has good capture ability and high simulation accuracy for large eddy and subgrid vortices in the wake area of wind turbine.

(2) Wind shear makes the attack angle of each section of the blade periodically deviate from the theoretical optimal design angle of attack, which leads to the decrease of wind turbine torque. 


\section{Acknowledgments}

The authors thank financial support from the Youth Program of National Natural Science Foundation of China (NSFC) under Grant No. 51908430.

\section{Reference}

1. Jia Y.Y., Chen J.J., Wang H.J. (2018) Research on Influence of Wind Wheel Elevation Angle on Wind Turbine Aerodynamic Performance. Acta Energiae Solaris Sinica, 39(04): 1135-1141.

2. Yu H.N., Zhu F.L., Liu Y. (2008) Overview of Aeroelastic Stability of Wind Turbines. Journal of Machine Design, (06):1-3.

3. Hansen M.O.L., SФRensen J.N., Voutsinas S., et al. (2006) State of the art in wind turbine aerodynamics and aeroelasticity. Progress in Aerospace Sciences, 42(4): 285-330.

4. Jonkman J., Butterfield S., Musial W., and Scott G. (2009) Definition of a 5-MW reference wind turbine for offshore system development. Office of Scientific \& Technical Information, Golden, Colorado.

5. He Y.L., Wang Y., Li Q. (2009) Lattice Boltzmann method: theory and applications. Beijing Science Press, Beijing.

6. Qian Y.H., D'Humières D., Lallemand P. (1992) Lattice BGK models for Navier-Stokes equation. Europhysics Letters, 17(6): 479-484.

7. Xu C., Huang H.Q., Shi C., et al. (2020) Numerical Simulation of Wind Turbine Wake Based on LBMLES Method under Typical Complex Terrain. Proceedings of the CSEE, (13).

8. Nicoud F., Ducros F. (1999) Subgrid-scale stress modelling based on the square of the velocity gradient tensor. Flow, Turbulence and Combustion, 62(3): 183-200. 